\title{
Neural-network-based sensor data fusion for multihole fluid velocity probes
}

\author{
Anindya Ghosh, David M. Birch and Olaf Marxen
}

\begin{abstract}
For measuring three components of velocity in unknown flow fields, multi-hole pressure probes possess a significant advantage. Unlike methods such as hot-wire anemometry, laser-Doppler velocimetry and particle-image velocimetry, multi-hole pressure probes can provide not only the three components of local velocity, but also static and stagnation pressures. However, multi-hole probes do require exhaustive calibration. The traditional technique for calibrating these probes is based on either look-up tables or polynomial curve fitting, but with the low cost and easy availability of powerful computing resources, neural networks are increasingly being used. Here, we explore the possibility to further reduce measurement uncertainty by implementing neural-networkbased methods that have not been previously used for probe calibration, including supervised and unsupervised learning neural networks, regression models and elastic-map methods. We demonstrate that calibrating probes in this way can reduce the uncertainty in flow angularity by as much as $50 \%$ compared to conventional techniques.
\end{abstract}

\section{Introduction}

$\mathrm{W}$ ITH the wide variety and availability of low-cost sensors, large distributed networks of sensors may now be routinely deployed. The critical limitation of these networks then becomes the calibration process: extracting the best possible approximations of the desired measurand from an overdefined system of imperfect sensors. This process of 'sensor data fusion' has already been extensively reviewed, with applications in wireless communications, navigation, target recognition and medical diagnostics $(1 ; 2)$.

One particular application that has seen some development in recent years has been the multihole velocity probean intrusive, pressure-based instrument which returns three components of fluid velocity based on multiple discrete pressure measurements taken on the surface of the probe tip. Since additional data about the fluid state are usually available as well, and since the pressure measurements themselves can be highly uncertain (or even independent of flow state under particular operating conditions), the reduction of data from the multihole velocity probe is a good candidate for sensor data fusion techniques. Indeed, there have already been several demonstrations of this $(3 ; 4)$. Here, the calibration data set will be extended to include direct measurements of fluid

A. Ghosh is with the Department of Mechanical Engineering, Indian Institute of Technology Bombay, Mumbai, India.

D. M. Birch and O. Marxen are with the Department of Mechanical Engineering Sciences, University of Surrey, Guildford, Surrey, GU2 7XH, UK; e-mail:d.birch@surrey.ac.uk. state parameters which may have an effect on the pressure sensors or their responses (including temperature, absolute pressure and humidity), and will be compared to a more conventional high-precision look-up table technique.

\section{A. Multi-hole velocity probes}

A multi-hole probe consists of a long sting with a tip machined usually either in the form of a hemisphere or truncated cone having some number $n$ pressure taps distributed around the surface of the tip. To return three components of velocity $u, v$ and $w$ (parallel to the $x, y$ and $z$ axes, respectively), then necessarily $n \geq 3$ (typically, $n=5$ or $n=7)$. To eliminate the velocity magnitude $U$ as an independent variable and reduce the calibration space from three dimensions to two, the pressures are normalized against some approximation of the local dynamic pressure $P_{d}=\rho U^{2} / 2$, where $\rho$ is the fluid density and is assumed to be constant. The error introduced by this approximation is reasonably independent of $U$ and may therefore be treated as just another empirical function obtained by calibration.

The number of independent variables may be further reduced if the geometry of the probe tip is prescribed: this was conventionally done due to limitations in computational resource for data reduction. The independent variables can then be taken as the differences and/or averages in normalized pressures between particular holes, as either will be uniquely related to the flow direction but may be discontinuous $(5 ; 6 ; 7)$. This, however, will necessarily increase uncertainty. As a baseline comparison, then, the technique of Shaw-Ward et al. (8) is adopted here as data from all holes are retained and the geometry of the probe is not prescribed. In this technique, a nondimensional pressure coefficient $C_{i}$ is defined for each $i$ th hole as

$$
C_{i}=\frac{\max \left(P_{i}\right)-P_{i}}{\max \left(P_{i}\right)-\min \left(P_{i}\right)}
$$

where $P_{i}$ is the dimensional pressure at the $i$ th hole. Note that $C_{i}$ is independent of $U$, and varies only with the flow angle. The stagnation pressure has been approximated as the maximum pressure recorded and the static pressure as the minimum. The difference $\max \left(P_{i}\right)-\min \left(P_{i}\right)$ is used to approximate the dynamic pressure, and the error of this approximation is quantified as the coefficient $C_{d}$ such that

$$
C_{d}=\frac{\frac{1}{2} \rho U^{2}}{\max \left(P_{i}\right)-\min \left(P_{i}\right)}
$$




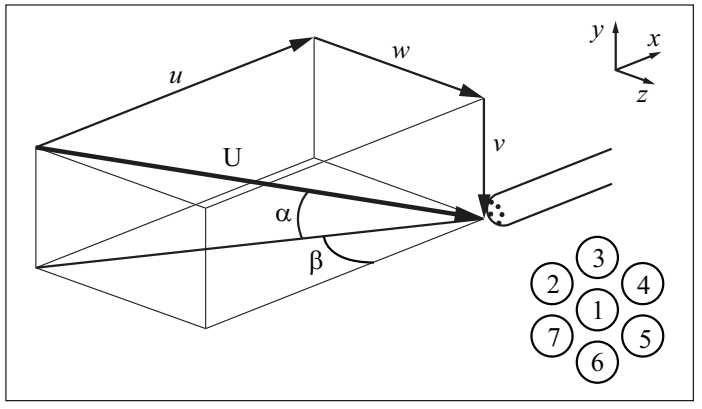

Fig. 1. Velocity components and flow angles relative to the probe axis, and indexing convention used for the seven-hole probe.

Then, the pitch angle $\alpha$ and yaw angle $\beta$ of the flow (as defined in Figure 1) and $C_{d}$ can be expressed as

$$
\begin{aligned}
\alpha & =f_{\alpha}\left(C_{1}, C_{2}, \ldots, C_{n}\right) \\
\beta & =f_{\beta}\left(C_{1}, C_{2}, \ldots, C_{n}\right) \\
C_{d} & =f_{d}(\alpha, \beta)
\end{aligned}
$$

where $f_{\alpha}, f_{\beta}$ and $f_{d}$ are empirical functions to be determined by calibration. During an experimental measurement, the $n$ pressures are converted into coefficients using eq. (1); $\alpha$ and $\beta$ are then obtained by interpolation from the empirical functions $f_{\alpha}$ and $f_{\beta}$. With the pitch and yaw angles obtained, $C_{d}$ can likewise be interpolated from $f_{d}$. The velocity magnitude may then be obtained from eq. (2), and the velocity components obtained from trigonometry (see (8) for a detailed description of the technique).

\section{B. Artificial neural networks and data reduction}

The probe calibration technique described above reduces to carrying out two $n$-dimensional interpolations and one two-dimensional one for each data point. This can be computationally intensive, especially given the higherorder interpolations generally required by the limited amount of calibration data which can be collected. Also, if any part of the probe tip is located in a region of separated wake flow (which can happen at large angles), data from some holes would no longer be independent and the uncertainty can become unacceptably high.

Because of their value in sensor data fusion applications, there has been some effort at implementing artificial neural networks (ANNs) to this problem: ANNs are much more tolerant of unknown mixes of high- and low-quality data, and can be trained to ignore information from pressure holes in areas of flow separation. This has been demonstrated on spherical probes having $n=12$ and $n=18$, which are particularly susceptible to separation effects (4). Neural networks also do not require any a priori assumptions about probe geometry, and have been shown to be more accurate and faster than conventional calibration techniques $(9 ; 3 ; 10)$.

More recently, techniques such as elastic maps and selforganising maps have been applied to data fusion problems in other applications, including bioinformatics, economics and political sciences $(11 ; 12)$, but have not yet been applied for calibration or uncertainty quantification for flow probes. The purpose of this work, therefore, is to compare the effectiveness of these and other ANN-based techniques in processing multihole probe data, together with more conventional regression models and nonlinear techniques including Monte-Carlo processes $(13 ; 14)$ and the conventional look-up table technique.

\section{Methodology}

\section{A. Experimental data}

For the purposes of comparing and assessing the different calibration techniques, data from the experiments of Shaw-Ward et al. (15) have been used. In these experiments, calibration data at known angles $(\alpha, \beta)$ were obtained using a hemispherical-tipped seven-hole probe (Surrey Sensors Ltd. series ID7HP-2K5) in a wind tunnel with uniform flow at a fixed speed $U=10 \mathrm{~m} / \mathrm{s}$. Data were obtained over a range $-40^{\circ} \leq \alpha \leq 40^{\circ}$ and $-40^{\circ} \leq \beta \leq 40^{\circ}$, with the (arbitrary) hole indexing convention as shown in Figure 1. The pitch and yaw angles had an estimated uncertainty of $\pm 0.2^{\circ}$. Additional measurements were collected at $(\alpha, \beta)=(0,0)$ over the range $0 \mathrm{~m} / \mathrm{s} \leq U \leq 15 \mathrm{~m} / \mathrm{s}$. A total of 6563 measurements were collected. Although the effects of Reynolds number and Mach number are not considered, these are unlikely to affect the overall outcomes $(16 ; 14 ; 3)$.

The probe used seven differential pressure sensors referenced directly the local static pressure, and had a full-scale range of $\pm 2.5 \mathrm{kPa}$ and an estimated uncertainty of less than $0.25 \%$ full-scale. The probe also measured local ambient temperature, absolute (static) pressure and humidity using co-located ancillary environmental sensors, as well as an internal system temperature (used for temperature corrections), all to within less than $\pm 0.5 \%$. Figures 2 (a) and (b) show contours of $C_{i}$ at the central hole and one of the peripheral holes, respectively. Although the ambient conditions were not controlled as part of the experiment, Figure 2 (c) shows how they changed in time. During the calibration process, measurements were collected at varying $\beta$ while at fixed $\alpha$, so that $\alpha$ increased approximately linearly with time. The full-scale variations in atmospheric temperature, pressure and relative humidity were $1.1^{\circ} \mathrm{C}$, $2.36 \mathrm{kPa}$ and $11.7 \%$, respectively.

\section{B. Sensor data fusion approaches}

Three different ANN-based methods have been used in order to invert eq. (3). In all cases, it was assumed that the sensor outputs satisfied the generalized responsesurface model for directional velocity probes to the sixth order (15). The calibrations were implemented using the MATLAB Statistics and Machine Learning Toolbox (release 2018a) on a laptop PC with an $2.60 \mathrm{GHz}$ i5 processor.

First, the Levenberg-Marquardt algorithm was implemented. In this damped least-squares approach, a twolayer feed-forward network was trained, yielding a shallow 

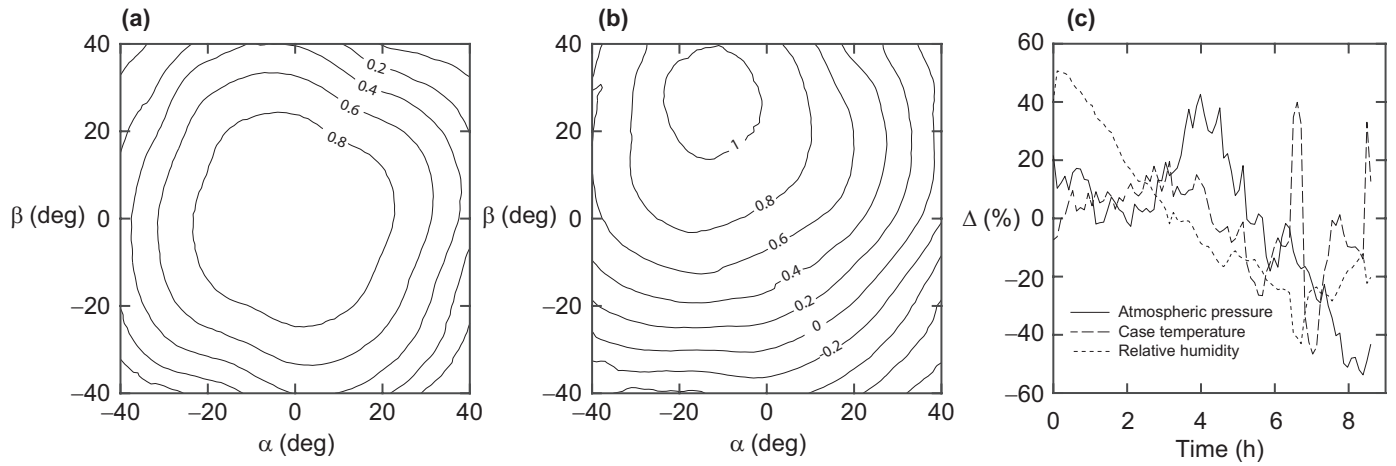

Fig. 2. Data from (15). (a), contours of $C_{i}$ from the central hole; (b) contours of $C_{i}$ from hole 4; (c) relative variation of ambient temperature, pressure and humidity as percent full-scale variation.

neural network with one input-hidden-output stack. The input layer used the 11 parameters measured by the probe, while the output layer had 3 output parameters $(\alpha, \beta$ and $U$ ). The number of neurons in the hidden layer was selected as that which yielded the lowest standard deviation in $U$. Although the ambient environmental conditions are not normally expected to affect the results (indeed, this is one of the operating requirements of the probe) this would highlight and eliminate any residual error from the temperature compensation process. The Levenberg-Marquardt algorithm was then used to obtain the minimum of the function $F(x)$, such that

$$
F(x)=\frac{1}{2} \sum f_{i}(x)^{2}
$$

where $f_{i}$ is the transfer function for each of the 11 input variables. To test this approach, $70 \%$ of the data set was selected to train the network and $15 \%$ of the data was used to validate the training, completing the calibration procedure. Then, the remaining $15 \%$ of the data set was used to assess the data conversion process by comparing the flow angle returned to the angle set during measurement.

Next, a regression learner was employed; this trains a regression model to predict data using supervised machine learning and requires both input and target vectors. Because of the formulation of the solver used, only one target vector could be implemented for all the input vectors. Therefore each of the three outputs were independently regressed to obtain the appropriate models to predict each target vector. Once the model training was complete, the root-mean-ssquare (RMS) error was obtained. A 10-fold cross-validation was used to prevent overfitting, in which the input and target data were randomly split into ten exclusive subsamples of equal size on which the individual regressions were performed. One of the subsamples was used as the validation dataset while the others were used as training data. This cross-validation was repeated ten times, and all of the subsamples were used as the validation dataset exactly once. The final regression was then taken as the average of the ten realizations. To reduce dimensionality, principal component analysis was also used (17). Note that although the technique used to assess these models necessarily had to be different from that used for the ANN, a holdout validation was also tested with $70 \%$ of data used for training, and there was no significant difference in the outcome.

A self-organizing map technique was implemented to characterize the input data by generating a topographic map. The mapping methods are a preliminary feature extraction step, resulting in data reduction as the relationships between all the input parameters are explored (18). A $27 \times 27$ grid was used, yielding 729 individual neurons. In this way, all 6561 data points are accurately clustered while not requiring a significant amount of computational resource. Although self-organizing maps provide low-dimensional data visualization and mapping which preserves the data topology, they often fail to correctly reflect the distances between the data on the low dimensional map, thus reducing the fidelity of the visualization. Since the response surface model of a directional velocity probe is known to be parabolic (15), features will tend to cluster together and the resolution of the mapping (and accuracy of the data conversion) will decrease based on defined initial conditions.

Elastic maps can be used to address this problem (12): they are known to provide a better representation of $3 \mathrm{D}$ principal manifolds than more conventional nonlinear fitting techniques, and are therefore able to provide better representations of the sensor response surfaces. This approach is analogous to mechanical springs bending and stretching between the nodes; the expectationmaximization algorithm minimizes the springs' total energy which guarantees a local minimum to effectively approximate non-linear principal manifolds. In this implementation, an elastic map was wrapped around a rigid $12 \times 12$ grid along the principal components; this grid size was selected in order to avoid overfitting. For this investigation, the elastic map tool ViDaExpert based on the technique of Zinovyev (12) was used.

For the purposes of comparison, the data reduction process was also compared against more conventional linear regression and ANN techniques already demonstrated $(3 ; 4 ; 14)$. 
A Monte-Carlo simulation was implemented for which the generalized response-surface model of (15) was used, in which $C_{i}(\alpha, \beta)$ is approximated as a sixth-order polynomial. The standard error of the velocity magnitude was determined over the entire calibration space given some number $N$ of evenly-spaced calibration measurements. The calibration measurements were taken as a subset of the data available, with $N=20,34,81,102,162,204,300$, 500, 850 and 6561 .

A simple linear regression model was also tested, approximating the response of the system as

$$
\mathbf{y}=\mathbf{B}_{\mathbf{0}}+\mathbf{X B}+\boldsymbol{\varepsilon}
$$

where $y$ is the model output, $\mathbf{X}$ is the dimensional observed predictor variable matrix (the seven port pressures, the absolute ambient pressure, the fluid and sensor temperatures and the humidity), $\mathbf{B}_{\mathbf{0}}$ is the intercept, $\mathbf{B}$ is a vector of the regression coefficients of the observed predictor variables and $\boldsymbol{\varepsilon}$ is Gaussian noise with zero mean and variance $\sigma^{2}$. All the regression models implemented provided coefficients that could be used to predict the velocities over the range $40^{\circ} \leq \alpha \leq 40^{\circ}, 40^{\circ} \leq \beta \leq 40^{\circ}$ given the same 11 predictor variables. Note that since the relationship between pressure and velocity is known to be parabolic, the dimensional pressures were raised to the power $1 / 2$ before being used in the linear regression model.

To investigate the model sensitivity to each of the different input parameters, a stepwise function was implemented. Each predictor variable was regressed, in turn, with the target variable. The $p$-values for an $F$-statistic were then obtained for each predictor variable. If the $p$ value was below a prescribed threshold of significance (arbitrarily set at 0.05 ), the predictor could be eliminated from the set (19).

Finally, a Markov chain Monte-Carlo (MCMC) method was used. This method can be used as a basis for the Gibbs sampler to generate a Markovian sequence such that the stationary distribution is the target distribution. Since the probe tip was hemispherical, a conjugate prior distribution was defined using the inviscid solution for the pressure distribution around a sphere, as

$$
C_{i}=\frac{9}{4} \theta_{i}-\frac{5}{4}
$$

where $\theta_{i}$ is the angle subtended between the position vector of the $i$ th hole in the probe tip and the incident flow angle. To implement the Gibbs sampling, for each draw a conjugate prior model $\mathbf{B}_{0}, \mathbf{B}_{j}, \sigma^{2} \mid \psi_{j}$ was created, where $\psi_{j}$ is the latent Gaussian scaling parameter of the $j$ th regression coefficient; sampling was then performed from the posterior of $\mathbf{B}_{0}, \mathbf{B}_{j}, \sigma^{2} \mid \psi_{j}, \mathbf{y}, \mathbf{X}$ using the current value of $\psi_{j}$ for each $j=1,2, \ldots, p$; finally, sampling was performed from $\psi_{j} \mid \mathbf{B}_{0}, \mathbf{B}_{j}, \sigma^{2}, \mathbf{y}, \mathbf{X}$ using the current values of $\mathbf{B}_{0}, \mathbf{B}_{j}$ and $\sigma^{2}$. Lasso estimates can be interpreted as posterior mode estimates when the regression parameters have independent and identical double-exponential priors (20). As a result, the Lasso estimate can be taken as

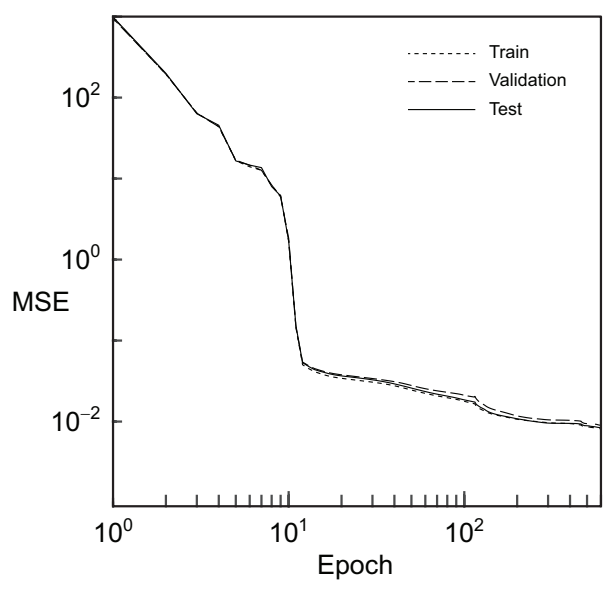

Fig. 3. Mean-square error as a function of number of epochs.

the mode of the posterior distribution of the B estimates obtained from the Gibbs sampling. This in turn allows Lasso regularization to be performed. However, conditioning on $\sigma^{2}$ is important as it ensures that the full posterior is unimodal; a lack of unimodality slows the convergence of the Gibbs sampler. Conditioning was therefore performed to increase convergence using the $\lambda$ regularization parameter.

\section{Results}

\section{A. Standard feedforward backpropagation network}

In the implementation of the standard feedforward backpropagation network trained with a LevenbergMarquardt algorithm, the mean-square error decreased rapidly with increasing number of hidden neurons; 11 hidden neurons were therefore used, yielding a meansquare error of $0.84 \%$.

Figure 3 shows the performance of the ANN at each epoch; the best validation was reached at 617 epochs, after which the untrained validation subset error rate begins to increase. The validation error is $0.89 \%$, while the test error was $0.84 \%$. By comparison, a generalized look-up calibration algorithm yields an overall uncertainty of $1.88 \%$ (15). The error between the output and the target vectors is Gaussian, with a mean of -0.0094 and $\sigma=0.0529$.

\section{B. Traditional machine learning regression approaches}

A variety of different regression approaches were tested, and the resultant mean-square errors are included in Table I. The lowest errors were found using the Gaussian process regressions (GPR) with an exponential kernel for $\alpha$ and $\beta$ and a Matern $5 / 2$ kernel for $U$. Note that predictions for each of $\alpha, \beta$ and $U$ were obtained individually with the 11 parameters, as a full 11-parameter regression was not possible with the available resource.

Figure 4 compares a selection of the outputs of $\alpha$ and $\beta$ returned by the exponential GPR to the actual angles set during the measurements (a limited range is displayed in 
TABLE I

Regression learner-generated models and their mean-square error.

\begin{tabular}{|c|c|c|c|c|}
\hline \multirow{3}{*}{ Model } & & \multicolumn{3}{|c|}{ Mean-square error } \\
\cline { 3 - 5 } Regression & Type & $U$ & $\alpha$ & $\beta$ \\
& Linear & 0.0295 & 2.085 & 8.407 \\
& Interactions & 0.0295 & 0.680 & 2.580 \\
\hline \multirow{2}{*}{ Stepwise } & Robust & 0.0295 & 2.687 & 9.271 \\
\hline \multirow{3}{*}{ Tree } & Stepwise & 0.0295 & 0.680 & 2.580 \\
\hline \multirow{5}{*}{ SVM } & Fine & 0.0369 & 0.738 & 0.930 \\
& Medium & 0.0298 & 1.326 & 1.642 \\
& Coarse & 0.0296 & 3.589 & 4.142 \\
& Linear & 0.0295 & 3.210 & 8.579 \\
& Quadratic & 0.0294 & 1.814 & 2.979 \\
& Cubic & 0.0293 & 3.363 & 2.964 \\
& Medium Gaussian & 0.0294 & 4.922 & 4.575 \\
& Coarse Gaussian & 0.0294 & 2.007 & 3.381 \\
Ensemble & Boosted Trees & 0.0295 & 2.640 & 3.241 \\
\hline \multirow{3}{*}{ GPR } & Bagged Trees & 0.0284 & 2.984 & 3.775 \\
& Squared Exponential & 0.0000 & 0.013 & 0.016 \\
& Matern 5/2 & 0.0000 & 0.010 & 0.012 \\
& Exponential & 0.0138 & 0.006 & 0.010 \\
& Rational Quadratic & 0.0000 & 0.012 & 0.016 \\
\hline
\end{tabular}

the figure in order to emphasize any differences). A very good agreement is achieved between these, as expected. In all cases, $U$ was returned to within less than $0.5 \%$ with one notable exception: the first and last data points collected with the probe as part of the calibration process were at $U=0$, in order to check for sensor drift. These data points are not normally used in calibration, but were retained here in order to test the robustness of the ANN technique, and returned the correct velocity to within $3.5 \%$ full-scale. Two scenarios were tested: one in which both $U=0$ points were used for training and the mean-square error (MSE) checked, and another in which one was used for training and the other for validation. Both scenarios gave almost identical MSE. Ideally, data would be present throughout arbitrary values of $U$ from 0 to $10 \mathrm{~m} / \mathrm{s}$. This is valid as physical reasons are present for the corresponding 11 parameters.

\section{Self-organizing map}

For the purposes of assessing the efficacy of the selforganizing map, a $27 \times 27$ neuron layer topology was created, as shown in Figure 5. The size of the filled hexagons indicate the number of training data points associated with each of the neurons, ranging from $n=2$ to $n=24$. The clustering of data associations around the centre and edges of the field is consistent with the response surface model for the probe, as shown in Figure 2.

Because the system had 11 degrees of freedom, it is difficult to graphically visualize all of the weightings in a meaningful way. Instead, the neighbour distances have been calculated and are shown in Figure 6. At the centre of the field, the distances between adjacent neurons are

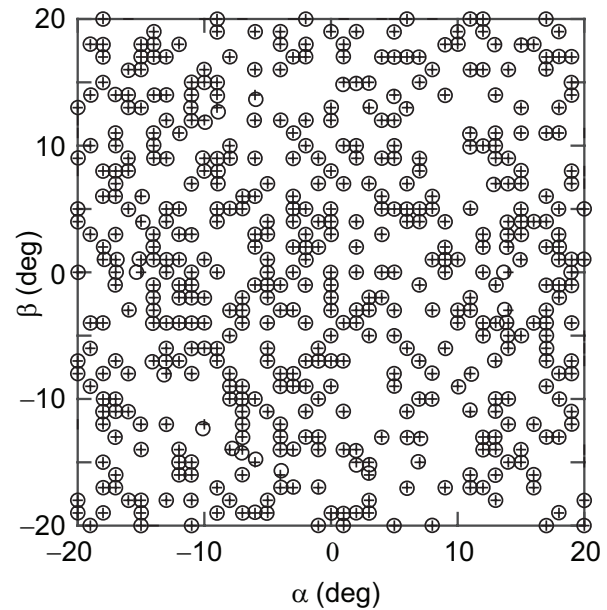

Fig. 4. Comparison between set and returned angles using the Exponential GPR regression. ॰, returned angles; +, set angles.

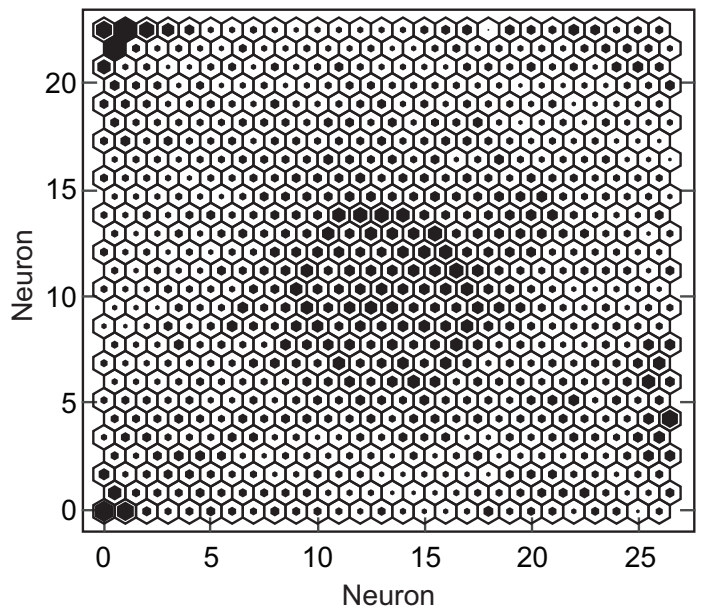

Fig. 5. Neuron locations in the hexagonal SOM topology, showing cluster centres. The fill of the individual hexagons correspond to number of input vectors in the cluster.

small and the weight vectors of adjacent neurons are closely spaced; the spacing increases toward the edges. Typical weight planes are also shown in fig. 7, for the same data shown in Figure 2. Data for only relative humidity appear to demonstrate a strong correlation with pitch angle, however since pitch also varied almost linearly with time, this is just indicative of the dominance of the slow ambient changes over the course of the measurements. A similar response was observed in the other environmental measurements (not shown).

\section{Elastic Map}

The regression coefficients for the elastic map are shown in table II; note that the $U=0$ zeroing data were included in the calibration data set. Estimates of velocity were obtained from the data set using the resultant calibrated regression model, and the mean square error between the actual and predicted outputs was $0.0165 \%$. This is a significant improvement on the mean-square error of $1.88 \%$ 


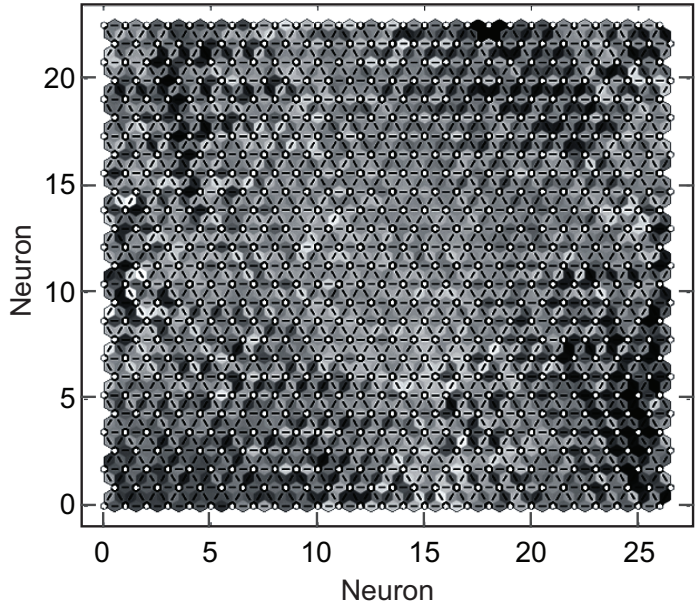

Fig. 6. U-matrix showing the SOM neighbor weight distances. Note that the localized saturation at the top of the plot is the result of a single bad point.

obtained using a polynomial fit and a 7-dimensional lookup table (8), and is significantly less than the reported uncertainty in the dependent variables: $(\alpha$ and $\beta$, for example, were accurate to within $\left.\pm 0.2^{\circ}\right)$.

E. Simple, step and Markov Chain Monte-Carlo regressions

For the purposes of comparison, a number of more conventional regression techniques were implemented as well. The simple regression model was found to have an adjusted $R^{2}=0.46$, suggesting that a simple linear model does not provide an accurate representation of the data. Similarly, the stepwise regression had an adjusted $R^{2}=0.82$. Although this is a significant improvement on the fit from linear regression, it still was not high enough for practical implementation. However, the stepwise regression can be considered as a reasonable approximation of the final model.

For the purposes of assessing MCMC sampling (Gibbs sampler) with Bayesian lasso regression, lasso plots were used to obtain the optimal value $\lambda$, the weight given to the regularisation term or $L^{1}$ norm), using elastic net regularization in the Gibbs sampler to optimize the weight of lasso ( $L^{1}$ norm) versus ridge $\left(L^{2}\right.$ norm). The lasso plots were then used to obtain the cross-validation deviance and quantify the effect of $\lambda$ on the lasso fit. With increasing $\lambda$, the regularization term increases and more of the coefficients become vanishingly small. In order to make the model more robust, a minimum crossvalidation error plus one standard deviation was used, yielding $\lambda=7.812 \times 10^{-4}$ : a value sufficiently small that all parameters were considered significant.

From the elastic net regularization, $\xi$ was varied such that $0.1 \leq \xi \leq 0.9$, and the value $\lambda=4.911 \times 10^{-5}$ was selected as the average of the coefficient vector of the 75 th iteration, as further iterations yielded no improvement and the effect of $\xi$ was insignificant. Trace plots of the
Gibbs sampler showed that the Markov chain Monte-Carlo sample was mixing well.

The posterior means of the regression coefficients and the disturbance variance (mean-square error) were then estimated. The first 5000 draws were treated as a burn-in and discarded. Histograms of the following posterior draws were very closely Gaussian, so the mean was considered as a good approximation of the mode. The corresponding value of $\lambda$ was computed, and then lasso regression was implemented using this $\lambda$. For $\sigma$, the posterior mean from the Gibbs sampler was used. The resultant regression coefficients are compared to simple linear regression (SLR) in table II. The mean square errors of all three models were small, demonstrating that all three models may be considered compliant. Moreover, the mean square errors of all the three models are lower than that obtained using a conventional look-up table calibration. The SLR takes 2.27 seconds, Lasso regression takes 46.4 seconds while the Gibbs sampler takes 46.2 seconds.

TABLE II

Comparison of all the estimates, including auto-zero points.

\begin{tabular}{|c|c|c|c|}
\hline Predictor & SLR & Lasso & Gibbs \\
\hline Intercept & -14.579 & -14.523 & -13.607 \\
$P_{1}(\times 100)$ & -2.7704 & -2.7696 & -2.7685 \\
$P_{2}(\times 100)$ & 0.6048 & 0.6044 & 0.6041 \\
$P_{3}(\times 100)$ & -0.3567 & -0.3563 & -0.3497 \\
$P_{4}(\times 100)$ & 0.6046 & 0.6052 & 0.6045 \\
$P_{5}(\times 100)$ & 1.3970 & 1.3956 & 1.3949 \\
$P_{6}(\times 100)$ & 1.0497 & 1.0504 & 1.0531 \\
$P_{7}(\times 100)$ & 1.0284 & 1.0280 & 1.0254 \\
$P_{\text {atm }}$ & 0.9473 & 0.9456 & 0.9166 \\
$T_{\text {atm }}$ & 1.7322 & 1.7322 & 1.7323 \\
$T_{1}$ & -1.8156 & -1.8155 & -1.8155 \\
$R H(\times 100)$ & -1.2551 & -1.2554 & -1.2554 \\
$\mathrm{MSE}(\times 100)$ & 1.5907 & 1.5877 & 1.6185 \\
\hline
\end{tabular}

IV. Resolution effects

\section{A. Response surface model}

Because all of the regression techniques used depended on the quality of the response surface model, this was assessed by means of a Monte-Carlo process. A normalized, robust bi-square method was used to model the calibration response surface with minimum standard error. Note that the fit was not significantly affected by outliers.

Following the recommendations of Shaw-Ward et al. (15), the response surfaces were modelled as sixth-order polynomials in two variables, resulting in $N=28$ terms; consequently, a minimum of 28 measurements are required in order to obtain a unique solution. Regression models were trained using sample sizes ranging from $0.25 N$ to $4 N$, with little difference in the convergence for $N>28$ (so that the inclusion of more data did not improve the accuracy). This is broadly consistent with the number of calibration points required if the locations of these are selected optimally (15). 
(a)

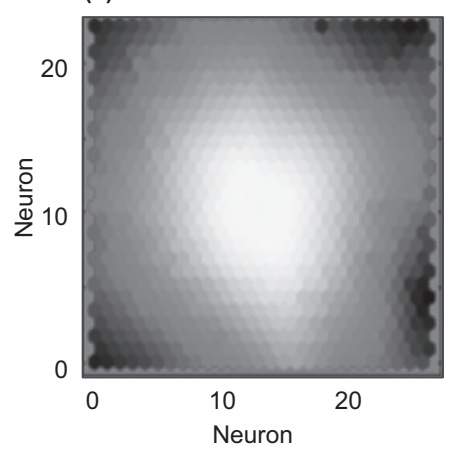

(b)

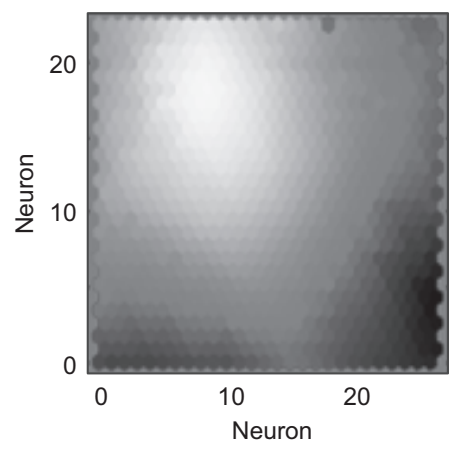

(c)

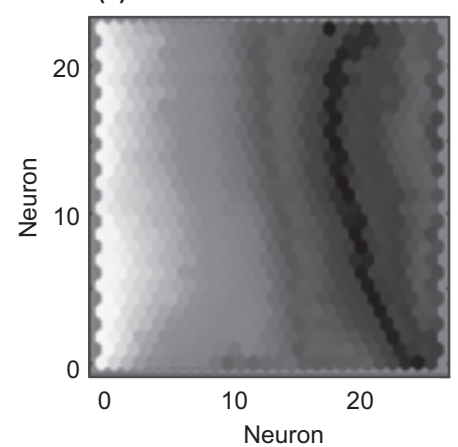

Fig. 7. SOM data weight planes for (a), the central hole pressure; (b), hole 4 pressure, and (c), relative humidity. Each weight plane defines weight of all clusters within each input parameter. Darker clusters indicate higher weights.

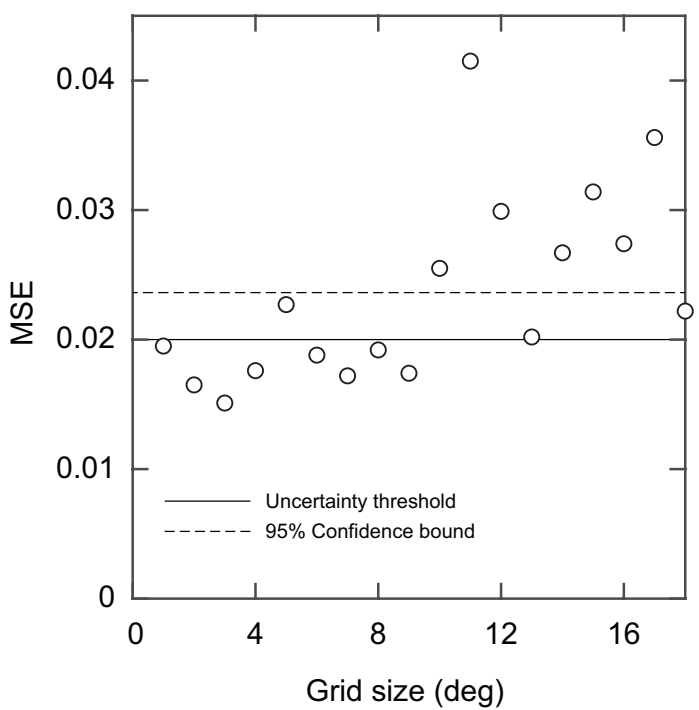

Fig. 8. MSE for various grid spacings. The MSE is consistently below the $95 \%$ Confidence bound for grid size below $10^{\circ}$.

\section{B. Effect of calibration grid resolution}

The experimental calibration measurements were resolved to $1^{\circ}$ in $\alpha$ and $\beta$. However, the intervals of the inputs used in the artificial neural networks could, of course, be freely chosen to yield maximum acceptable mean-square error. Figure 8 shows the normalized mean square error as a function of the selected step size used for training the ANN. The minimum MSE was found to be $1.5 \%$ at a grid resolution of $3^{\circ}$. If an acceptable MSE is defined as $2 \%$ (which is within the expected angular sensitivity of a multi-hole probe), the measurements do not begin to demonstrate unacceptable levels of MSE until the grid size exceeds $10^{\circ}$ : this is consistent with earlier experimental observations (10).

The effect of calibration range was also assessed by further limiting the data sets used for training the ANN to $|\alpha|$ and $|\beta|<10^{\circ}, 20^{\circ}, 30^{\circ}$ and $40^{\circ}$. Taking the maximum allowable MSE again as $2 \%$, maximum grid spacings of $10^{\circ}, 8^{\circ}, 9^{\circ}$ and $11^{\circ}$ were obtained for for these ranges, respectively. It is important to note that the data do not extend to angles were large areas of separation would be expected over the holes- this would, of course, increase the required resolution to achieve the target maximum MSE.

\section{Conclusions}

Conventional techniques for processing data from multihole probes were developed under the constraint of severely limited computer power. With this constraint now largely eliminated, it becomes useful to reassess these techniques. Recent advances in sensor data fusion provide a framework with which to process multi-hole probe data in a more generalized way; equally, these allow ancillary measurements, such as the ambient environmental state, to be included within the calibration data set.

Several artificial neural network and machine learning tools have been applied to the problem of obtaining three components of velocity from red multi-hole probe measurements. Of all the methods tested, the elastic map approach yielded the lowest mean squared error. Although the regression learner approach using a Gaussian process regression achieved a similar mean square error, it is not a composite model encompassing $\alpha, \beta$ and $U$ and is more computationally intensive relative to the other methods. A Monte-Carlo process was able to model the response surface with reasonable accuracy (with applied Gaussian noise), but was also computationally intensive.

Regression, classification and clustering neural networkbased approaches to address higher-order terms were also implemented. The use of SLR suggested low linearity of the dataset due to the low $\mathrm{R} 2$ value. The stepwise regression allowed the importance of the individual parameters to the regression red to be assessed based on their $p$-value before using MCMC sampling.

Focusing on non-linear algorithms, the regression learner was accurate within the explicit context of the dataset. The classification Levenberg-Marquardt algorithm proved to be accurate as well. The use of PCAenabled SOM and elastic map clustering methods allowed the visualization of the 11-dimensional dataset as a $2 \mathrm{D}$ space. Weight planes gave insights into which clusters are the most contributing. By preserving the topological 
structure, these techniques work as optimization methods and the regressions exhibit less error. Since elastic maps better maintain 3D principal manifolds, this technique proved to be the most accurate.

An elastic map-based approach was applied to implement the 3D manifold as a 2D topology, and it provided correlations between the input and output parameters. The results agreed well with the physical fluid flow phenomena, although some spurious correlations were obtained as a result of drift.

Overall, the techniques explored here typically provided uncertainties significantly lower than those obtained using the latest high-accuracy generalized calibration technique (15).

\section{Acknowledgments}

The authors are very grateful to Dr. Mark Hansen for his input, as well as to Surrey Sensors Ltd. for its support.

\section{References}

[1] D. Hall and J. Llinas, "An introduction to multisensor data fusion," Proc. IEEE, vol. 85, no. 1, pp. 6-23, 1997.

[2] B. Khaleghi, A. Khamis, F. Karray, and S. Razavi, "Multisensor data fusion: A review of the state-ofthe-art," Info. Fusion, vol. 14, pp. 28-44, 2013.

[3] L. Jeyan, J. Muruga, and M. Kumar, "Performance evaluation for multi-hole probe with the aid of artificial neural network," J. Theo. \& Appl. Information Tech., vol. 65, no. 3, 2014.

[4] O. Rediniotis and R. Vijayagopal, "Miniature multihole pressure probes and their neural-network-based calibration," AIAA J., vol. 37, no. 6, pp. 666-674, 1999.

[5] R. W. Gallington, "Measurement of very large flow angles with non-nulling seven-hole probes," USAFA Aeronautics Digest, vol. USAFA-TR-80-17, pp. 6088, DoI 10.1007/BF00 261 328, 1980.

[6] G. G. Zilliac, "Modelling, calibration, and error analysis of seven-hole pressure probes." Exp. Fluids, vol. 14, no. 1, pp. 104-120, 1993.

[7] A. J. Pisasale and N. A. Ahmed, "Development of a functional relationship between port pressures and flow properties for the calirbation and application of multihole probes to highly three-dimensional flows," Experiments in Fluids, vol. 36, pp. 422-436, 2004.

[8] S. Shaw-Ward, A. Titchmarsh, and D. M. Birch, "Calibration and use of n-hole velocity probes," AIAA Journal, vol. 53, no. 2, pp. 336-346, DoI 10.2514/1.J053 130, 2015.

[9] R. Benay, "A global method of data reduction applied to seven-hole probes," Exp. Fluids, vol. 54, no. 6, p. $1535,2013$.

[10] D. Sumner, "A comparison of data-reduction methods for a seven-hole probe," Journal of Fluids Engineering, vol. 124, pp. 523-527, 2002.

[11] M. Chacon, M. Levano, H. Allende, and H. Nowak, "Detection of gene expressions in microarrays by applying iteratively elastic neural net," Adaptive and Natural Computing Algorithms, pt 2, vol. 4432, p. $355,2007$.

[12] A. Zinovyev, "Data visualization in political and social sciences," in International Encyclopedia of Political Science, B. Badie, Berg-Schlosser, D., and L. A. Morlino, Eds. SAGE, 2011.

[13] Ö. Kisi, "River flow modeling using artificial neural networks," J. Hydrol. Eng., vol. 9, no. 1, pp. 60-63, 2004.

[14] M. Hölle, C. Bartsch, and P. Jeschke, "Evaluation of measurement uncertainties for pneumatic multihole probes using a Monte Carlo method," J. Eng. Gas Turb. \& Power, vol. 139, no. 7, p. 072605, 2017.

[15] S. Shaw-Ward, S. C. McParlin, P. Nathan, and D. M. Birch, "Optimal calibration of directional velocity probes," AIAA J., vol. 56, no. 7, pp. 2594-2603, 2017.

[16] D. Telionis, Y. Yang, and O. Rediniotis, "Recent developments in multi-hole probe (MHP) technology," 20th Intl. Congress of Mech. Eng., vol. 21, 2009.

[17] I. Jolliffe, Principal Component Analysis (2nd Ed.). Springer, 2002.

[18] I. Nabney, NETLAB (3rd Ed.). Springer, 2004.

[19] R. Fisher, The Design of Experiments (9th Ed.). Macmillan, 1971.

[20] R. Tibshirani, "Regression shrinkage and selection via the lasso," J. Royal. Statist. Soc B., vol. 58, no. 1, pp. 267-288, 1996 . 